\title{
ON THE DEGREE OF CONVERGENCE OF THE DEVELOPMENT
}

\section{OF A CONTINUOUS FUNCTION ACCORDING TO}

\author{
LEGENDRE'S POLYNOMIALS*
}

BY

\section{DUNHAM JACKSON}

It is the purpose of this paper to demonstrate three theorems and two corollaries, whose formal statement will be preceded by a brief explanation. The treatment is suggested by recent papers of LeBESGUE, $\dagger$ in which corresponding problems are discussed for the case of trigonometric series. A part of the work of LeBesGue is reproduced at length in my thesis, $\ddagger$ and where the developments of the present paper are closely parallel to those in the trigonometric case I shall occasionally refer the reader to one of those other presentations.

\section{I.}

Let $\omega(\delta)$ be a positive function of the positive variable $\delta$, which has the property that as $\delta$ increases, $\omega(\delta)$ never decreases, and $\omega(\delta) / \delta$ never increases, while $\lim _{\delta=0} \omega(\delta)=0$. Let $f(x)$ be a function of $x$, defined and continuous in the interval $-1 \leqq x \leqq 1$. Let $r_{n}(x)$ denote the difference

$$
\sum_{m=0}^{n} a_{m} P_{m}(x)-f(x),
$$

where the functions $P_{m}(x)$ are the polynomials of LEGENDRE, and

$$
a_{m}=\frac{2 m+1}{2} \int_{-1}^{1} f(x) P_{m}(x) d x .
$$

* Presented to the Society, February 24, 1912.

†Lebesgue, Annales de la Faculté de Toulouse, series 3, vol. 1 (1909), pp. 25-117. Referred to hereafter as LebesGUe I.

- Bulletin de la Soc. Math. de France, vol. 38 (1910), pp. 184-210. Referred to as Lebesgue II.

$\ddagger \ddot{U b e r}$ die Genauigkeit der Annäherung stetiger Funktionen durch ganze rationale Funktionen gegebenen Grades und trigonometrische Summen gegebener Ordnung. Dissertation, Göttingen, 1911. Referred to as Thesis. 
It will be convenient to use a notation of LANDAU* expressing the asymptotic relation of two functions $g_{1}(n), g_{2}(n)$, of a variable $n$, as $n$ becomes infinite, $g_{2}(n)$ being supposed positive for all values of $n$ from some point on. The expression $g_{1}(n)=O\left(g_{2}(n)\right)$ means that the ratio $g_{1}(n) / g_{2}(n)$ remains finite as $n$ becomes infinite, and the expression $g_{1}(n)=o\left(g_{2}(n)\right)$ means that $\lim _{n=\infty} g_{1}(n) / g_{2}(n)=0$.

Then the following is true for any value of $x$ interior to the interval $(-1,1)$, and uniformly throughout any closed interval interior to that interval:

Theorem I. If $f(x)$ satisfies the condition that

$$
\left|f\left(x_{2}\right)-f\left(x_{1}\right)\right| \leqq \omega(\delta)
$$

whenever $x_{1}$ and $x_{2}$ are any two points of the closed interval $(-1,1)$ such that

then $\dagger$

$$
\left|x_{2}-x_{1}\right| \leqq \delta
$$

$$
\left|r_{n}(x)\right|=O\left(\omega\left(\frac{1}{n}\right) \log n\right)
$$

A special case of this is the

Corollary. If $\lim _{\delta=0} \omega(\delta) \log \delta=0$, the development of $f(x)$ according to Legendre's polynomials is convergent. $\ddagger$.

This statement also holds for any interior point of the interval $(-1,1)$ and uniformly in any closed interval interior to that interval. As to the endpoints $+1,-1$, no conclusion is drawn.

To prove Theorem I, I shall make use of a proposition established in my thesis, $\S$ which, when $\omega(\delta)$ is restricted as above, amounts to a statement that $f(x)$ can be approximately represented throughout the interval by a polynomial of degree $n$ or lower, with a maximum error not exceeding a constant multiple of $\omega(1 / n)$, for all values of $n$. This being granted, a process of

* Landau, Handbuch der Lehre von der Verteilung der Primzahlen, Leipzig and Berlin, 1909, pp. 31, 59, 61. LEBEsGue uses occasionally a notation which looks similar to this, with quite a different meaning.

† Cf. Lebesgue II, pp. 199-202 of the volume, 16-19 of the article. By saying that such a relation as this "holds uniformly in an interval," I mean, of course, that the ratio of the left-hand to the right-hand member, regarded as a function of two variables, remains finite for all values of $n$ from a certain point on and all values of $x$ in the interval.

$\ddagger$ A somewhat similar condition for convergence, which however does nct include the present one and is not included in it, is given by DINI, Serie di Fourier e altre rappresentazioni analitiche delle funzioni di una variabile reale, Pisa, 1880 . See pp. 281-282, $\$ 116$; in $\$ 72$, to which the reader is referred back, sec especially condition $(b)$.

\& Thesis, Satz V, p. 40. The polynomials there called $P_{n}(x)$ have nothing to do with the polynomials of LEGENDRE. A simplified proof of Satz IV of the thesis, which is used in establishing Satz V, is given in another article by the author presently to appear in these Transactions, On approximation by trigonometric sums and polynomials. Another interesting special case of the present theorem is that of the LIPscHITz condition, $\omega(\delta)=$ constant $\times \delta$. 
reasoning which is readily transferred from the trigonometric case to the present one reduces our problem to that of showing that the absolute value of the remainder $r_{n}(x)$, formed for any continuous function $f(x)$ whose absolute value does not exceed 1 in the interval $-1 \leqq x \leqq 1$, can never be greater than a constant multiple of $\log n$, as long as $x$ is restricted to an interval $(-1+\epsilon, 1-\epsilon), \epsilon>0$. The essential argument leading to this simplification of the problem is that the $n$th partial sum of the development of a polynomial of the $n$th degree according to the functions $P_{m}(x)$ is identical with the polynomial itself. More simply still, it is seen by writing down at length the expression for this partial sum that it is sufficient to show that, if

where

$$
I_{n}(x)=\int_{-1}^{1}\left|K_{n}(x, t)\right| d t
$$

then

$$
K_{n}(x, t)=\sum_{m=0}^{n} \frac{2 m+1}{2} P_{m}(x) P_{m}(t),
$$

$$
I_{n}(x)=O(\log n)
$$

uniformly in any interval $(-1+\epsilon, 1-\epsilon), \epsilon>0$. $^{*}$ The notation $K_{n}$ is adopted from a recent paper of $\mathrm{HAAR}_{\mathrm{A}} \dagger$ to the first pages of which $\mathrm{I}$ shall have occasion to refer.

From the recursion formula for $P_{n}(x)$ follows readily the identity $\ddagger$

$$
K_{n}(x, t)=\frac{n+1}{2} \frac{P_{n+1}(x) P_{n}(t)-P_{n}(x) P_{n+1}(t)}{x-t} .
$$

We shall use also the asymptotic formula

$$
P_{n}(x)=\sqrt{\frac{2}{n \pi \sin \theta}}\left[\cos \left(\left(n+\frac{1}{2}\right) \theta-\frac{\pi}{4}\right)+\frac{\rho_{n}(\theta)}{n}\right],
$$

where $x$ is set equal to $\cos \theta, \pi \geqq \theta \geqq 0$, and $\rho_{n}(\theta)$ is a function which remains below a certain constant in absolute value for all values of $n$, and all values of $x$ in the interval $\left(-1+\frac{1}{2} \epsilon, 1-\frac{1}{2} \epsilon\right.$ ); it is assumed that $\epsilon$ is held fast. It follows at once that in this interval $\left|P_{n}(x)\right|$ never exceeds a constant multiple of $1 / v$, let us say

$$
\left|P_{n}(x)\right| \leqq \frac{H}{v^{\prime} n}
$$

* Cf. Lebesque I, pp. 116-117; Lebesgue II, pp. 196-198, 201, of the volume, 13-15, 18, of the article; Thesis, pp. 49-50.

† HAAR, Rendicontidel Circolo Matematico di Palermo, vol. 32 (1911), pp. 132-142; pp. 132-135.

‡ Christofrel, Crelle's Journal, vol. 55 (1858), pp. 61-82; p. 73. 
It is further pointed out by HAAR that*

$$
\int_{-1}^{1}\left|P_{n}(x)\right| d x=O(1 / \sqrt{n}) .
$$

As the proposition to be proved will not be affected by neglecting a finite number of values of $n$, let us suppose $n$ so large that $1 / n<\epsilon / 2$. Then the points $x-1 / n, x+1 / n$ surely lie inside the interval $\left(-1+\frac{1}{2} \epsilon, 1-\frac{1}{2} \epsilon\right)$, if, as we shall suppose throughout, $x$ is a point of the interval $(-1+\epsilon, 1-\epsilon)$. The integral $I_{n}(x)$ may be broken up into five parts,

$$
\begin{aligned}
\int_{-1}^{1}\left|K_{n}(x, t)\right| d t & =\int_{-1}^{-1+\epsilon / 2}+\int_{-1+e / 2}^{x-1 / n}+\int_{x-1 / n}^{x+1 / n}+\int_{x+1 / n}^{1-\ell / 2}+\int_{1-\ell / 2}^{1} \\
& =I_{n}^{\prime}+I_{n}^{\prime \prime}+I_{n}^{\prime \prime \prime}+I_{n}^{(I V)}+I_{n}^{(V)} .
\end{aligned}
$$

In $I_{n}^{\prime}$ and $I_{n}^{(V)}, t$ takes on only such values that $|x-t| \geqq \frac{1}{2} \epsilon$, and so, by (1),

Hence

$$
\left|K_{n}(x, t)\right| \leqq \frac{n+1}{\epsilon}\left|P_{n+1}(x) P_{n}(t)-P_{n}(x) P_{n+1}(t)\right| .
$$

$I_{n}^{\prime} \leqq \frac{n+1}{\epsilon}\left[\left|P_{n+1}(x)\right| \int_{-1}^{-1+\epsilon}\left|P_{n}(t)\right| d t+\left|P_{n}(x)\right| \int_{-1}^{-1+e}\left|P_{n+1}(t)\right| d t\right]$.

Since $P_{n}(x)=O(1 / \sqrt{n})$ and $P_{n+1}(x)=O(1 / \sqrt{n})$, uniformly for $-1+\epsilon \leqq x \leqq 1-\epsilon$, and

$$
\int_{-1}^{-1+e}\left|P_{n}(t)\right| d t<\int_{-1}^{1}\left|P_{n}(t)\right| d t=O(1 / \sqrt{n}),
$$

and similarly for the integral of $\left|P_{n+1}(t)\right|$, it follows that there exists a constant which $I_{n}^{\prime}$ never exceeds. The same is true of $I_{n}^{(\text {P) }}$.

Proceeding to the second of the five integrals, we find that

$$
\begin{array}{r}
I_{n}^{\prime \prime} \leqq \frac{n+1}{2}\left[\left|P_{n+1}(x)\right| \int_{-1+\mathrm{e} / 2}^{x-1 / n}\left|\frac{P_{n}(t)}{x-t}\right| d t+\left|P_{n}(x)\right| \int_{-1+\mathrm{e} / 2}^{x-1 / n}\left|\frac{P_{n+1}(t)}{x-t}\right| d t\right] \\
\leqq \frac{n+1}{2}\left[\frac{2 H^{2}}{n} \int_{-1+\mathrm{e} / 2}^{x-1 / n} \frac{d t}{x-t}\right]=H^{2} \frac{n+1}{n} \int_{1 / n}^{x+1-\ell / 2} \frac{d u}{u} \\
<H^{2} \frac{n+1}{n} \int_{1 / n}^{2} \frac{d u}{u}=H^{2} \frac{n+1}{n}(\log 2+\log n) .
\end{array}
$$

* Since

and

$$
\int_{-1}^{1}\left[P_{n}(x)\right]^{2} d x=\frac{2}{2 n+1},
$$

by ScrWarz's inequality.

$$
\int_{-1}^{1}\left|P_{n}(x)\right| d x \leqq \sqrt{2 \int_{-1}^{1}\left[P_{n}(x)\right]^{2} d x}
$$


This remains inferior to a constant multiple of $\log n$. Similar reasoning applies to $I_{n}^{(I V)}$.

There remains only the third of the integrals. To handle this, we abandon the formula (1) and write $K_{n}(x, t)$ at length,

$$
\left|K_{n}(x, t)\right|=\left|\sum_{m=0}^{n} \frac{2 m+1}{2} P_{m}(x) P_{m}(t)\right| \leqq \sum_{m=0}^{n}\left|\frac{2 m+1}{2} P_{m}(x) P_{m}(t)\right|
$$

In any one term of this sum of absolute values, $\left|P_{m}(x)\right| \leqq H / \sqrt{m}$, and, for values of $t$ in the interval of integration, $\left|P_{m}(t)\right| \leqq H \mid \sqrt{m}$, and so the terms individually remain inferior to a suitably chosen constant.* The sum of the $n+1$ terms does not exceed a constant multiple of $n$. Therefore the integral of the sum, over an interval of length $2 / n$, remains inferior to a constant.

Putting these results together, we see that it is in fact true that $I_{n}(x)=O(\log n)$, uniformly in the interval $(-1+\epsilon, 1-\epsilon) \cdot \dagger$ This result, combined with Theorem $\mathrm{V}$ of $\mathrm{my}$ thesis, as has been indicated above, establishes Theorem I of the present paper.

Another proposition demonstrated in my thesis $\ddagger$ is that if $f(x)$ is a function possessing a $(k-1)$ th derivative $f^{(k-1)}(x)$ which satisfies a Lipschitz condition,

$$
\left|f^{(k-1)}\left(x_{2}\right)-f^{(k-1)}\left(x_{1}\right)\right| \leqq \lambda\left|x_{2}-x_{1}\right|,
$$

in some interval, $\lambda$ being a constant, then $f(x)$ may be approximately represented in that interval by a polynomial of the $n$th degree or lower, for all values of $n$, with a maximum error not exceeding a constant multiple of $1 / n^{k}$. This may be combined with the relation $I_{n}(x)=O(\log n)$ to establish the correctness of the following statement, which applies to any point, or uniformly to any closed interval, interior to $(-1,1)$ :

Theorem II. If $f(x)$ is a function possessing a $(k-1)$ th derivative which satisfies a Lipschitz condition throughout the closed interval $(-1,1)$, then, for this function $f(x)$,

$$
\left|r_{n}(x)\right|=O\left(\frac{\log n}{n^{k}}\right)
$$

A precisely analogous result for the case of Fourier's series§ may be de-

* This last statement is of course true also of the term $\frac{1}{2} P_{0}(x) P_{0}(t)$, to which the previous reasoning, with the asymptotic formula, does not apply.

† The formal work above is a modification of that of HAAR in the article cited; but he does not have occasion to obtain the relation $I_{n}(x)=O(\log n)$.

† Thesis, Satz II, p. 23.

8 For the case $k=1$, this theorem is to be found in the article referred to as LEBEsGUE II, pp. 199-201 of the volume, 16-18 of the article, as a part of the analogon of Theorem I above; a result closely parallel to, but neither including nor included in, that for the case $k=2$ of 
duced immediately from Satz VII (page 46) and Hilfssatz II (page 49) of my thesis.

II.

The following theorem is in a way complementary to the first; it shows that our first theorem proves as much as can be proved without making other hypotheses than those we have employed. Let $\omega(\delta), f(x), r_{n}(x)$ have the meaning given them at the beginning of $\mathrm{I}$. that

THEOREM III. There exists a function $f(x)$ which satisfies the condition

$$
\left|f\left(x_{2}\right)-f\left(x_{1}\right)\right| \leqq \omega(\delta)
$$

whenever $x_{1}$ and $x_{2}$ are any two points of the closed interval $(-1,1)$ such that

$$
\left|x_{2}-x_{1}\right| \leqq \delta
$$

while there is an interior point of the interval at which*

$$
r_{n}(x) \neq o(\omega(1 / n) \log n) .
$$

For $\omega(\delta)=1 /|\log \delta|$ this reduces to the

Corollary. There exists a function $f(x)$ for which $\omega(\delta) \log \delta$ remains finite as $\delta$ approaches zero, $\dagger$ while the expansion of $f(x)$ according to Legendre's polynomials diverges at an inner point of the interval $(-1,1)$.

(It can not, however, diverge to infinity, by virtue of Theorem I.)

We are to construct a function $f(x)$, satisfying the condition stated in the theorem, for which we can show that for an interior point of the interval $(-1,1)$, and for infinitely many values of the integral index $n, r_{n}(x)$ is in absolute value greater than $\omega(1 / n) \log n$ multiplied by a constant independent of $n$. We may confine our attention entirely to one point $x$, and we may leave out of consideration as many values of $n$ as we please, finite or infinite in number, provided only that we still have infinitely many values left. Let us restrict ourselves to the point $x=0$, and to values of $n$ which are odd and of the form $4 n^{\prime}+3$. A number of other conditions will successively be imposed on $n$, but they will all be satisfied by choosing $n$ sufficiently large, and so will be consistent with each other and with the one already introduced.

the present theorem is stated in an article by WeyL, Rendiconti del Circolo Matematico di Palermo, vol. 32 (1911), pp. 118-131; p. 128, footnote. See also the author's article $O$ n approximation, etc., referred to in a previous footnote.

* Cf. Lebesgue II, pp. 202-206 of the volume, 19-23 of the article.

tOf course $f(x)$ will then be continuous. 
For any function $f(x)$, keeping the notation previously used,

$$
\sum_{m=0}^{n} a_{m} P_{m}(x)=\int_{-1}^{1} f(t) K_{n}(x, t) d t .
$$

Since $P_{n}(0)=0$ when $n$ is odd, we see from (1) that, for $n$ odd,

accordingly

$$
K_{n}(0, t)=-\frac{n+1}{2} P_{n+1}(0) \frac{P_{n}(t)}{t} ;
$$

$$
\sum_{m=0}^{n} a_{m} P_{m}(0)=-\frac{n+1}{2} P_{n+1}(0) \int_{-1}^{1} \frac{f(t) P_{n}(t)}{t} d t .
$$

Let us set

$$
x=\cos \theta, \quad \pi \geqq \theta \geqq 0,
$$

as was done before in formula (2), and let $g$ be a constant, which will surely exist, such that $\left|\rho_{n}(\theta)\right| \leqq g$ for all values of $n$ and for $\frac{1}{6} \pi \leqq \theta \leqq \frac{6}{6} \pi$.

Since we suppose $n+1$ divisible by 4 , it follows that $\frac{1}{2}(n+1) \pi$ is a multiple of $2 \pi$, and

$$
\begin{aligned}
\cos \left[\left(n+\frac{1}{2}\right) \theta-\frac{\pi}{4}\right] & =\cos \left[\left(n+\frac{1}{2}\right) \theta-\frac{\pi}{4}-(n+1) \frac{\pi}{2}\right] \\
& =\cos \left[\left(n+\frac{1}{2}\right)\left(\theta-\frac{\pi}{2}\right)-\frac{\pi}{2}\right] \\
& =\sin \left(n+\frac{1}{2}\right)\left(\theta-\frac{\pi}{2}\right),
\end{aligned}
$$

or, in terms of a new angle

$$
\begin{gathered}
\varphi=\frac{\theta}{2}-\frac{\pi}{4}, \quad \frac{\pi}{4} \geqq \varphi \geqq-\frac{\pi}{4}, \\
\cos \left[\left(n+\frac{1}{2}\right) \theta-\frac{\pi}{4}\right]=\sin (2 n+1) \varphi .
\end{gathered}
$$

Let $\beta$ be a number satisfying the inequalities $-\frac{1}{2}<\beta<0$; $\beta$ may be any such number, but when once chosen is to be held fast. If $x=\beta$, then $\theta=\cos ^{-1} \beta$ lies between $\frac{2}{3} \pi$ and $\frac{1}{2} \pi$, and $\varphi=\frac{1}{2} \cos ^{-1} \beta-\frac{1}{4} \pi$ lies between $\pi / 12$ and 0 . Let this value of $\varphi$ be denoted by $\alpha$.

Let $n$ be so large that $3 \pi /(2 n+1) \leqq \alpha$, and let $p$ be the largest integer for which $p \pi /(2 n+1) \leqq \alpha$, accordingly $p \geqq 3$. By making $n$ sufficiently large, and hence $g / n \geqq\left|\rho_{n}(\theta)\right| / n$ sufficiently small, we can make the roots of $P_{n}(x)$ in the interval $\frac{6}{6} \pi \geqq \theta \geqq \frac{1}{6} \pi,-\frac{1}{2} \sqrt{3} \leqq x \leqq \frac{1}{2} \sqrt{3}$, correspond so closely to roots of $\sin (2 n+1) \varphi$ that $P_{n}(x)$ vanishes for a value of $\varphi$, 
say $\varphi=\sigma$, which is nearer to $\pi /(2 n+1)$ than to any other root of $\sin (2 n+1) \varphi$, and for a value $\varphi=\tau$ nearer to $(p+1) \pi /(2 n+1)$ than to any other root of $\sin (2 n+1) \varphi$, and similarly for each of the intervening roots of $\sin (2 n+1) \varphi$. Let the values of $x$ corresponding to $\sigma$ and $\tau$ be denoted by $\gamma$ and $\delta$ respectively. The latter numbers will surely be interior to the interval $\left(-\frac{1}{2}, 0\right)$, for large values of $n$, and we will assume $n$ taken so large that this is the case. Furthermore, $\frac{1}{2} \pi /(2 n+1)<\sigma<2 \pi /(2 n+1)$, and $\tau>p \pi /(2 n+1)>\sigma$.

We are now ready to define an auxiliary function $\chi_{n}(x, \beta)$, analogous to the function $\chi_{n}(x, \alpha)$ defined on p. 58 of my thesis, following the example of Lebesgue. Let

$$
\begin{array}{lrl}
\chi_{n}(x, \beta) & =0, & -1 \leqq x \leqq \delta ; \\
\chi_{n}(x, \beta)=\frac{1}{\sqrt{n}} P_{n}(x), & \delta \leqq x \leqq \gamma ; \\
\chi_{n}(x, \beta)=0, & \gamma \leqq x \leqq 1
\end{array}
$$

This is surely a continuous function of $x$. The formula

$$
\left(x^{2}-1\right) \frac{d}{d x} P_{n}(x)=n x P_{n}(x)-n P_{n-1}(x),
$$

together with the fact that, for the values of $x$ that come into consideration, $P_{n}(\dot{x})$ never surpasses a constant multiple of $1 / \sqrt{n}$, shows that $\chi_{n}(x, \beta)$ satisfies a Lipschitz condition with respect to $x$,

$$
\left|\chi_{n}\left(x_{2}, \beta\right)-\chi_{n}\left(x_{1}, \beta\right)\right| \leqq \Lambda\left|x_{2}-x_{1}\right|,
$$

where, as long as $\beta$ lies in the interval $\left(-\frac{1}{2}, 1\right), \Lambda$ is a constant independent of $n$ and $\beta$ as well as of $x_{1}$ and $x_{2}$.

If we denote now by $a_{m}$ the coefficient of $P_{m}(x)$ in the expansion of $\chi_{n}(x, \beta)$, and by $r_{n}(x)$ the corresponding remainder

$$
\sum_{m=0}^{n} a_{m} P_{m}(x)-\chi_{n}(x, \beta)
$$

(the index of $r_{n}$ is intentionally set equal to that of $\chi_{n}$ ), then we have by (3), noticing that $\chi_{n}(0, \beta)=0$,

$$
\left|r_{n}(0)\right|=\left|\frac{n+1}{2} \frac{P_{n+1}(0)}{\sqrt{n}} \int_{\delta}^{\gamma} \frac{\left[P_{n}(t)\right]^{2}}{t} d t\right|
$$

Now let $t=\cos \theta, \varphi=\frac{1}{2} \theta-\frac{1}{4} \pi$, the determination of $\theta$ and $\varphi$ being rendered 
unique as before by the conventions $\pi \geqq \theta \geqq 0, \frac{1}{4} \pi \geqq \varphi \geqq-\frac{1}{4} \pi$.

$$
\begin{aligned}
{\left[P_{n}(t)\right]^{2} } & =\frac{2}{n \pi \sin \theta}\left[\sin (2 n+1) \varphi+\frac{\rho_{n}(\theta)}{n}\right]^{2} \\
& =\frac{2}{n \pi \sin \theta}\left[\sin ^{2}(2 n+1) \varphi+\frac{\bar{\rho}_{n}(\theta)}{n}\right],
\end{aligned}
$$

where $\bar{\rho}_{n}$, like $\rho_{n}$, is a function which remains finite for all values of $n$ and $\theta$ in question.

$$
\begin{aligned}
& t=\cos \theta=\cos \left[\frac{\pi}{2}+2 \varphi\right]=-\sin 2 \varphi, \quad d t=-\sin \theta d \theta=-2 \sin \theta d \varphi \\
& \int_{\delta}^{\gamma} \frac{\left[P_{n}(t)\right]^{2}}{t} d t=-\int_{\sigma}^{\tau} \frac{2}{n \pi \sin \theta} \cdot \frac{\sin ^{2}(2 n+1) \varphi}{\sin 2 \varphi} \cdot 2 \sin \theta d \varphi \\
&-\int_{\sigma}^{\tau} \frac{2}{n \pi \sin \theta} \cdot \frac{\bar{\rho}_{n}(\theta)}{n} \cdot \frac{1}{\sin 2 \varphi} \cdot 2 \sin \theta d \varphi .
\end{aligned}
$$

To form an idea of the magnitude of the latter integral, let us take into account that in the integrand, $\varphi \geqq \sigma, 2 \varphi \geqq 2 \sigma>\pi /(2 n+1)$, from which it follows that the greatest value attained by $1 / \sin 2 \varphi$ within the limits of integration is in order of magnitude $O(n)$. As $\sin \theta$ cancels from numerator and denominator, we see that

and

$$
\int_{\sigma}^{r} \frac{2}{n \pi \sin \theta} \cdot \frac{\bar{\rho}_{n}(\theta)}{n} \cdot \frac{1}{\sin 2 \varphi} \cdot 2 \sin \theta d \varphi=O\left(\frac{1}{n}\right)
$$

$$
\int_{\delta}^{\gamma} \frac{\left[P_{n}(t)\right]^{2}}{t} d t=-\frac{4}{n \pi} \int_{\sigma}^{\tau} \frac{\sin ^{2}(2 n+1) \varphi}{\sin 2 \varphi} d \varphi-O\left(\frac{1}{n}\right)
$$

Consider now the integral

$$
\int_{\sigma}^{\tau} \frac{\sin ^{2}(2 n+1) \varphi}{\sin 2 \varphi} d \varphi .
$$

Since within the limits of integration $0<\varphi<\pi / 12$, the integrand is always positive; furthermore $\sin 2 \varphi=2 \sin \varphi \cos \varphi<2 \sin \varphi$; and $\sigma<2 \pi /(2 n+1)$, $\tau>p \pi /(2 n+1)$. Hence

$$
\begin{aligned}
& \int_{\sigma}^{\tau} \frac{\sin ^{2}(2 n+1) \varphi}{\sin 2 \varphi} d \varphi>\int_{2 \pi /(2 n+1)}^{p \pi /(2 n+1)} \frac{\sin ^{2}(2 n+1) \varphi}{\sin 2 \varphi} d \varphi \\
&>\frac{1}{2} \int_{2 \pi /(2 n+1)}^{p \pi /(2 n+1)} \frac{\sin ^{2}(2 n+1) \varphi}{\sin \varphi} d \varphi .
\end{aligned}
$$


The last integral is shown on p. 60 of my thesis to be greater than

$$
\frac{1}{6}[\log (p+1)-\log 3]=\frac{1}{6} \log (p+1)\left[1-\frac{\log 3}{\log (p+1)}\right] \text {. }
$$

From the way in which $p$ was chosen,

$$
\frac{(p+1) \pi}{2 n+1}>\alpha, \quad p+1>\frac{2 \alpha}{\pi}\left(n+\frac{1}{2}\right),
$$

and if $n$ is sufficiently large,

also

$$
\log (p+1)>\log \frac{2 \alpha}{\pi}+\log \left(n+\frac{1}{2}\right)>\frac{1}{2} \log n,
$$

$$
1-\frac{\log 3}{\log (p+1)}>\frac{1}{2}
$$

These inequalities being satisfied, we have

$$
\int_{\sigma}^{\tau} \frac{\sin ^{2}(2 n+1) \varphi}{\sin 2 \varphi} d \varphi>\frac{1}{48} \log n .
$$

In the expression for $\left|r_{n}(0)\right|, P_{n+1}(0)$ is this only factor for the magnitude of which a lower limit has not been ascertained. Remembering that we have assumed $n$ to be of the form $4 n^{\prime}+3$, we readily find from the asymptotic expression for $P_{n}(x)$ that

$$
P_{n+1}(0)=\sqrt{\frac{2}{(n+1) \pi}}\left[1+\frac{\rho_{n+1}(\pi / 2)}{n}\right],
$$

which for sufficiently large values of $n$ is greater than a constant positive multiple of $1 / \sqrt{n}$. Putting the various factors together, we see finally that there exists a positive constant $l$, independent of $n$ (and also of $\alpha$ ), such that from a certain point on*

$$
\left|r_{n}(0)\right|>l \frac{\log n}{n}
$$

For any one function $\chi_{n}(x, \beta)$, however, this inequality has been established only for the particular value of $n$ for which the function was formed.

One further observation concerning the functions $\chi_{n}(x, \beta)$ will be of importance. It is clear from the definition of the functions and the asymptotic expression for $P_{n}(x)$ that there exists a constant $M$ such that for all values of $n, \beta$, and $x$, satisfying the conditions that we have imposed,

$$
\frac{2 n+1}{2 \pi} \chi_{n}(x, \beta)<M \text {. }
$$

* The point at which this inequality begins to be true may to be sure depend on $\alpha$, but that is immaterial. 
Still following the procedure of Lebesgue, we go on to define a single function $f(x)$ which satisfies a relation analogous to (5) for infinitely many values of $n$.

Let $\beta_{1}$ be a number satisfying the inequalities that have been imposed on $\beta$. Let $n_{1}$ be a value of $n$ so large that a function $\chi_{n_{1}}\left(x, \beta_{1}\right)$ can be defined and the subsequent work carried through as above. For a reason which will appear later, we shall also assume explicitly that $n_{1}$ is chosen so large that $g / n_{1}<1 / 10$, where $g$, as before, denotes a constant not less than the greatest value of $\left|\rho_{n}(\theta)\right|$ in the interval $\frac{1}{6} \pi \leqq \theta \leqq \frac{5}{6} \pi$. From now on the number $n_{1}$ so chosen shall be held fast. Let $\beta_{2}$ be a number, still negative, but greater than the upper limit of the interval of values of $x$ in which $\chi_{n_{1}}\left(x, \beta_{1}\right)$ takes on values different from zero. Let $n_{2}$ be a value of $n$ greater than $n_{1}$, and so large

(a) that the function $\chi_{n_{2}}\left(x, \beta_{2}\right)$ may be defined and used as above;

(b) that the interval in which $\chi_{n_{2}}\left(x, \beta_{2}\right)$ takes on values different from zero does not trespass upon the corresponding interval for $\chi_{n_{1}}\left(x, \beta_{1}\right)$;

(c) that the error of the partial sum, to terms of degree $n_{1}$, of the expansion in series of Legendre's polynomials of a function which nowhere exceeds $M \omega\left[2 \pi /\left(2 n_{2}+1\right)\right]$ in absolute value can not be greater numerically at any point than

$$
\frac{l}{4} \cdot \frac{2 n_{1}+1}{2 \pi} \cdot \omega\left(\frac{2 \pi}{2 n_{1}+1}\right) \cdot \frac{\log n_{1}}{n_{1}}
$$

The constants $M$ and $l$ have already been defined. It is readily seen that the last requirement can be fulfilled, as well as the others, since $n_{1}$ is fixed and $\omega\left[2 \pi /\left(2 n_{2}+1\right)\right]$ approaches zero as $n_{2}$ becomes infinite. Then let $\beta_{3}$ and $n_{3}$ be similarly chosen with respect to $\beta_{2}$ and $n_{2}$, and the corresponding function $\chi_{n_{3}}\left(x, \beta_{3}\right)$ constructed, and so on.

If $v_{1}, v_{2}, v_{3}, \cdots$ are constants, each of which is equal to 0 or 1 , the series

$$
f(x)=\frac{1}{4 \Lambda} \sum_{j=1}^{\infty} v_{j} \frac{2 n_{j}+1}{2 \pi} \omega\left(\frac{2 \pi}{2 n_{j}+1}\right) \chi_{n_{j}}\left(x, \beta_{j}\right),
$$

where $\Lambda$ is the constant of the relation (4), converges for all values of $x$ in the interval $-1 \leqq x \leqq 1$, and the function $f(x)$ so defined is continuous throughout the interval. This appears at once from the structure of the series, without the application of any general theorems, since at any one point not more than one term of the series is different from zero.

If $i$ is any positive integer, we may break up $f(x)$ into three parts: first, the sum of the first $i-1$ terms of the series; second, the $i$ th term; and third, the sum of the terms from the $(i+1)$ th on. Suppose these three parts, which are of course continuous functions, expanded in serit of Legendre's poly- 
nomials to terms of degree $n_{i}$, and let the remainders in these expansions at the point $x=0$ be denoted by $R_{1}, R_{2}, R_{3}$, respectively. In order to make our function $f(x)$ completely determinate, we still have to fix the coefficients $v_{i}$, about which we have said nothing except that each shall be equal to 0 or 1 . We will suppose $v_{1}=1$, and the others chosen successively so that, for each value of $i$,

$$
\left|R_{1}+R_{2}\right|>\frac{l}{2} \cdot \frac{1}{4 \Lambda} \cdot \frac{2 n_{i}+1}{2 \pi} \cdot \omega\left(\frac{2 \pi}{2 n_{i}+1}\right) \cdot \frac{\log n_{i}}{n_{i}} .
$$

If $\left|R_{1}\right|$ alone satisfies this inequality, we make $v_{i}=0$, and so $R_{2}=0$; if not, we make $v_{i}=1$, and then the inequality is surely satisfied, in consequence of (5). The third part of $f(x)$ nowhere exceeds $\frac{1}{4}(M / \Lambda) \omega\left(2 \pi /\left(2 n_{i+1}+1\right)\right)$ in absolute value, and so

$$
\left|R_{3}\right| \leqq \frac{l}{4} \cdot \frac{1}{4 \Lambda} \cdot \frac{2 n_{i}+1}{2 \pi} \cdot \omega\left(\frac{2 \pi}{2 n_{i}+1}\right) \cdot \frac{\log n_{i}}{n_{i}},
$$

since we have supposed $n_{i+1}$ chosen so large that this conclusion may be drawn.* Consequently $\dagger$

$$
\begin{aligned}
\left|R_{1}+R_{2}+R_{3}\right| & >\frac{l}{4} \cdot \frac{1}{4 \Lambda} \cdot \frac{2 n_{i}+1}{2 \pi} \cdot \omega\left(\frac{2 \pi}{2 n_{i}+1}\right) \cdot \frac{\log n_{i}}{n_{i}} \\
& \geqq \frac{l}{16 \Lambda \pi} \cdot \log n_{i} \cdot \omega\left(\frac{1}{n_{i}}\right) .
\end{aligned}
$$

This inequality, holding for the infinitely many values $n_{i}$ of $n$, shows that for the function $f(x)$ the reminder $r_{n}(0)$ is not $o(\omega(1 / n) \log n)$.

It remains to be shown that

$$
\left|f\left(x_{2}\right)-f\left(x_{1}\right)\right| \leqq \omega\left(\left|x_{2}-x_{1}\right|\right),
$$

where $x_{1}, x_{2}$, are any two different points of the interval $(-1,1)$.

We may distinguish two possibilities as to the position of these points: both may belong to a single interval throughout which $f(x)$ has a single definition as a constant times one of Legendre's polynomials, or they may not both belong to such an interval. The former case is the only one that requires extended consideration. If we can show that in that case

$$
\left|f\left(x_{2}\right)-f\left(x_{1}\right)\right| \leqq \frac{1}{2} \omega\left(\left|x_{2}-x_{1}\right|\right),
$$

the inequality $(6)$ will hold in all cases. $\ddagger$

* See $(c)$ above.

†Cf. Thesis, pp. 62-64, 67, 68-69.

$\ddagger$ Cf. Thesis, p. 68. 
Let us suppose, then, that $x_{1}$ and $x_{2}$ are two points of an interval throughuot which the equation holds:

$$
f(x)=\frac{1}{4 \Lambda} \cdot \frac{2 n+1}{2 \pi} \omega\left(\frac{2 \pi}{2 n+1}\right) \cdot \frac{1}{\sqrt{n}} P_{n}(x),
$$

where $n$ is some fixed integer, all the other terms of the series for $f(x)$ being identically zero in the interval. As will presently appear, it is easy to show that (7) holds provided that the, values $x_{1}$ and $x_{2}$ are sufficiently close to each other. But this in itself is not sufficient for our purpose. In the case of trigonometric series* it was possible to find points $\bar{x}_{1}$ and $\bar{x}_{2}$ at which the function in question takes on the same values as at $x_{1}$ and $x_{2}$ respectively, while $\bar{x}_{1}$ and $\bar{x}_{2}$ are so near together that the relation (7) can be obtained at once. In the present case it is no longer obvious that we can find such points, for the arches of the curve $y=f(x)$ in the interval under consideration are now not all of the same height, and a value attained on one arch is not necessarily attained on every other arch. It is possible to modify the reasoning, however, so that it is still effective. In the first place, if the difference $\left|x_{2}-x_{1}\right|$ is not greater than $2 \pi /(2 n+1)$, we set $\bar{x}_{1}=x_{1}, \bar{x}_{2}=x_{2}$. In the contrary case, the argument is as follows: We have supposed $n_{1}$, and hence each of the other indices $n_{i}$, chosen so large that for the values of $x$ with which we are concerned $\left|\rho_{n}(\theta)\right| / n \leqq g / n \leqq 1 / 10$, and for these values of $x$ it is always true furthermore that $\frac{1}{2} \leqq \sin \theta \leqq 1$. Consequently the absolute value of the factor

$$
\sqrt{\frac{1}{\sin \theta}}\left[\cos \left(\left(n+\frac{1}{2}\right) \theta-\frac{\pi}{4}\right)+\frac{\rho_{n}(\theta)}{n}\right],
$$

which can be taken out of the expression (2) for $P_{n}(x)$, is never greater than $\sqrt{2}(1+1 / 10)<1.6$, and is greater than $1-1 / 10=0.9$, which is more than half of 1.6, at every maximum or minimum of the cosine. This function (8), which needs only to be multiplied by a constant in order to be identical with $f(x)$ in the interval under consideration, takes on at every point of the interval where the cosine is equal to +1 a value greater than half its greatest positive value, and similarly for negative values. If $y$ is any value of $f(x)$ in the interval, $f(x)$ takes on the value $\frac{1}{2} y$ in the smaller interval between a maximum and an adjacent minimum of the cosine. Such an interval is described while $\theta$ varies through $\pi /\left(n+\frac{1}{2}\right)=2 \pi /(2 n+1)$, and $x=\cos \theta$ consequently varies through not more than the same amount, $2 \pi /(2 n+1)$. That is, returning to the points $x_{1}, x_{2}$, it is possible to find two values $\bar{x}_{1}, \bar{x}_{2}$, in an interval such as has just been described, so that

$$
f\left(\bar{x}_{1}\right)=\frac{1}{2} f\left(x_{1}\right), \quad f\left(\bar{x}_{2}\right)=\frac{1}{2} f\left(x_{2}\right) .
$$

* Cf. Thesis, pp. 67-68. 
In any case, then, there will be points $\bar{x}_{1}, \bar{x}_{2}$, such that the following inequalities are satisfied:

$$
\begin{gathered}
\left|f\left(\bar{x}_{2}\right)-f\left(\bar{x}_{1}\right)\right| \geqq \frac{1}{2}\left|f\left(x_{2}\right)-f\left(x_{1}\right)\right|, \\
\left|\bar{x}_{2}-\bar{x}_{1}\right| \leqq \frac{2 \pi}{2 n+1}, \\
\left|\bar{x}_{2}-\bar{x}_{1}\right| \leqq\left|x_{2}-x_{1}\right| .
\end{gathered}
$$

The rest of the argument offers nothing new in comparison with the trigonometric case. We know that

conseciuently

$$
\left|\chi_{n}\left(\bar{x}_{2}, \beta\right)-\chi_{n}\left(\bar{x}_{1}, \beta\right)\right| \leqq \Lambda\left|\bar{x}_{2}-\bar{x}_{1}\right| \text {, }
$$

$$
\left|f\left(\bar{x}_{2}\right)-f\left(\bar{x}_{1}\right)\right| \leqq \frac{1}{4} \cdot \frac{2 n+1}{2 \pi} \cdot \omega\left(\frac{2 \pi}{2 n+1}\right) \cdot\left|\bar{x}_{2}-\bar{x}_{1}\right| \leqq \frac{1}{4} \omega\left(\left|\bar{x}_{2}-\bar{x}_{1}\right|\right),
$$

the last inequality resulting from (10) and the fact that $\omega(\delta) / \delta$ is a never increasing function. Applying (9) and (11), and the fact that $\omega(\delta)$ never decreases as $\delta$ increases, we infer hence that

$$
\left|f\left(x_{2}\right)-f\left(x_{1}\right)\right| \leqq \frac{1}{2} \omega\left(\left|\bar{x}_{2}-\bar{x}_{1}\right|\right) \leqq \frac{1}{2} \omega\left(\left|x_{2}-x_{1}\right|\right) ;
$$

this completes the proof of Theorem III with its corollary.

\section{HARVARD UNIVERSITY,}

Cambridge, Mass. 